PHYSICAL REVIEW E 75, 060101(R) (2007)

\title{
Characterizing potentials by a generalized Boltzmann factor
}

\author{
V. Blickle, ${ }^{1}$ T. Speck, ${ }^{2}$ U. Seifert, ${ }^{2}$ and C. Bechinger ${ }^{1}$ \\ 12. Physikalisches Institut, Universität Stuttgart, Pfaffenwaldring 57, 70550 Stuttgart, Germany \\ ${ }^{2}$ II. Institut für Theoretische Physik, Universität Stuttgart, Pfaffenwaldring 57, 70550 Stuttgart, Germany
}

(Received 26 February 2007; published 1 June 2007)

\begin{abstract}
Based on the concept of a nonequilibrium steady state, we present a method to experimentally determine energy landscapes acting on colloidal systems. By measuring the stationary probability distribution and the current in the system, we explore potential landscapes with barriers up to several hundred $k_{\mathrm{B}} T$. As an illustration, we use this approach to measure the effective diffusion coefficient of a colloidal particle moving in a tilted potential.
\end{abstract}

DOI: 10.1103/PhysRevE.75.060101

PACS number(s): 05.40.-a, 82.70.Dd

Introduction. The interaction of soft matter systems with potential landscapes created by optical tweezers plays a key role in, e.g., mechanical flexibility measurements of single biomolecules or molecular motors [1,2], guiding of neuronal cells [3], or phase transitions of colloidal monolayers on patterned substrates $[4,5]$. In addition, extended optical lattices can be used as sorters for microscopic particles [6] or as microoptomechanical devices such as Couette rheometers [7]. Currently, no theories are available which can be used to directly calculate optical trapping forces on macromolecules. Thus the precise calibration of optical forces is a central issue in many experiments.

The simplest method to determine an optical potential $V(\mathbf{r})$ is to measure the equilibrium distribution $p_{\text {eq }}(\mathbf{r})$ of a highly diluted colloidal system at position $\mathbf{r}$. From the inverted Boltzmann factor

$$
V(\mathbf{r})=-k_{\mathrm{B}} T \ln p_{\mathrm{eq}}(\mathbf{r}),
$$

one directly obtains the underlying potential $V(\mathbf{r})$ with a typical energy resolution on the order of $0.1 k_{\mathrm{B}} T[4,8]$, where $k_{\mathrm{B}}$ is Boltzmann's constant and $T$ the temperature of the surrounding fluid. This technique, however, is only applicable to potential depths up to $\simeq 7 k_{\mathrm{B}} T$ which are effectively sampled by Brownian particles in equilibrium. For larger trapping potentials, optical forces are typically calibrated indirectly by taking advantage of Stokes law which relates the particle velocity to the friction force exerted by the surrounding solvent molecules. Accordingly, from the drift velocity of a particle, the underlying potential can be reconstructed [9-11]. Alternatively, within the drag force method, $V(\mathbf{r})$ can be determined from the particle's displacement upon moving the sample stage (and thus the liquid) with known velocity $[1,2,12,13]$. In contrast to Eq. (1), however, the latter two nonequilibrium methods neglect thermal fluctuations since only mean values of particle velocities or displacements are considered. While such fluctuations can be neglected at large trapping forces, this is no longer justified for external forces with strengths comparable to those exerted by fluctuating Brownian forces.

In this paper, we introduce a potential reconstruction method based on a generalization of Eq. (1) to nonequilibrium conditions. This is experimentally realized by generating a nonequilibrium steady state (NESS) for a colloidal par- ticle in a one-dimensional (toroidal) potential landscape. By measuring the stationary probability distribution and the current in the system, we can reliably calibrate potential wells between a few tens up to several hundreds of $k_{\mathrm{B}} T$. As a demonstration, we apply this method to the problem of giant diffusion where precise knowledge of the potential allows for a quantitative match of the experimental data with theoretical predictions.

Potential reconstruction. Our method is based on a generalization of the Boltzmann factor inversion (1) to nonequilibrium. The effectively one-dimensional motion of the particle along a toroidal trap is governed by a Langevin equation

$$
\dot{x}=\gamma^{-1} F(x)+\zeta(t),
$$

with $x$ the spatial coordinate and $\zeta(t)$ representing thermal noise with correlations $\left\langle\zeta(t) \zeta\left(t^{\prime}\right)\right\rangle=2\left(k_{\mathrm{B}} T / \gamma\right) \delta\left(t-t^{\prime}\right)$, where $\gamma=\frac{1}{3 \pi \eta d}$ is the friction coefficient, $d$ the particle diameter, and $\eta$ the solvent viscosity. The force $F(x)=-V^{\prime}(x)+f$ exerted on the particle stems from two sources: the gradient of the periodic potential $V(x+L)=V(x)$ and a nonconservative driving force $f$.

We define a "pseudopotential" $\phi(x)$ by writing the nonequilibrium steady-state probability distribution as $p_{\mathrm{s}}(x)$ $=\exp [-\phi(x)]$ resembling the Boltzmann factor. The stationary probability current through the toroid is given as

$$
j_{\mathrm{s}}=\gamma^{-1}\left[F(x) p_{\mathrm{s}}(x)-k_{\mathrm{B}} T p_{\mathrm{s}}^{\prime}(x)\right],
$$

which is constant in one dimension. We introduce the local mean velocity

$$
v_{\mathrm{s}}(x)=j_{\mathrm{s}} / p_{\mathrm{s}}(x)
$$

and obtain [14]

$$
\gamma v_{\mathrm{s}}(x)=F(x)+k_{\mathrm{B}} T \phi^{\prime}(x)=-V^{\prime}(x)+f+k_{\mathrm{B}} T \phi^{\prime}(x) .
$$

Integration of Eq. (4) leads to the potential

$$
V(x)=k_{\mathrm{B}} T \phi(x)+\int_{0}^{x}\left[f-\gamma v_{\mathrm{s}}(x)\right] d x
$$

up to an irrelevant additive constant. Using the definitions of $\phi(x)$ and $v_{\mathrm{s}}(x)$ we finally arrive at 


$$
V(x)=-k_{\mathrm{B}} T \ln p_{\mathrm{s}}(x)+f x-\gamma j_{\mathrm{s}} \int_{0}^{x} p_{\mathrm{s}}^{-1}(x) d x .
$$

Hence, the stationary probability $p_{\mathrm{s}}(x)$ and the local mean velocity $v_{\mathrm{s}}(x)$ determine the potential $V(x)$. The driving force $f$ can be determined by setting $x=L$ in Eq. (5) and using the periodicity of the potentials $V(x)$ and $\phi(x)$ as

$$
f=\frac{\gamma}{L} \int_{0}^{L} v_{\mathrm{s}}(x) d x .
$$

In thermal equilibrium both $j_{\mathrm{s}}$ and $f$ vanish and Eq. (6) reduces to the inverted Boltzmann factor, Eq. (1). Therefore, Eq. (6) can be understood as an extension of the Boltzmann factor to nonequilibrium stationary states.

Experiment. For an experiment exploiting Eq. (6), we use a scanning optical tweezers setup as described in detail elsewhere [15]. A laser beam $(\lambda \simeq 532 \mathrm{~nm})$ is deflected on a pair of galvanometric mirrors and focused with a $100 \times$, numerical aperture $(\mathrm{NA})=1.3$ oil immersion objective from below onto a silica bead immersed in water (diameter $d$ $\simeq 1.85 \mu \mathrm{m}$ ). Upon periodic modulation of the angular mirror positions we obtain a three-dimensional toroidal laser trap with a torus radius of $R \simeq 3.95 \mu \mathrm{m}$. At our driving frequencies $\nu_{\mathrm{T}} \simeq 100 \mathrm{~Hz}$, the particle cannot directly follow the rotating laser trap. Instead, every time the particle is passed by the laser tweezers, it experiences a minute kick along the rotation direction whose strength depends on the laser intensity $I_{0}[11]$. Because the particle trajectory is monitored with video microscopy at a sampling rate of $20 \mathrm{~Hz}$, single kicking events are not resolved and the driving force $f$ along the angular direction can be considered as constant [15]. In addition, the intensity of the laser is weakly modulated along the toroidal trap. This is achieved with an electro-optical modulator (EOM) being controlled by a function generator which is synchronized with the scanning motion of the mirrors. This intensity modulation $I_{m}(x)$ leads to a periodic potential $V(x)$ with $x$ the arclength coordinate along the circumference of the torus. It has been demonstrated that the resulting optical forces of such an intensity-modulated scanned laser tweezers exerted on a colloidal particle correspond to those of a tilted periodic potential [15].

To experimentally demonstrate that $V(x)$ can be obtained under nonequilibrium steady-state conditions, the intensity of the scanned laser tweezer along the toroidal trap was varied according to

$$
I(x)=I_{0}+I_{m} \sin \left(n \frac{2 \pi x}{L}\right) \quad(n=4) .
$$

Figure 1 shows the steady-state probability distribution $p_{\mathrm{s}}(x)$ as obtained from the particle trajectory and the corresponding pseudopotential $\phi(x)$ for $I_{0}=44 \mathrm{~mW}$ and $I_{m}=10 \mathrm{~mW}$. Together with the driving force $f=34 k_{\mathrm{B}} T / \mu \mathrm{m}$ as determined from the measured local mean velocity $v_{\mathrm{s}}(x)$ [cf. Eq. (7)], we finally arrive at the potential $V(x)$ which is also plotted in Fig. 1 as a dotted line. Clearly, under NESS conditions the minima and maxima of $\phi(x)$ and $V(x)$ do not coincide. In addition, $\phi(x)$ varies in a less pronounced way than $V(x)$

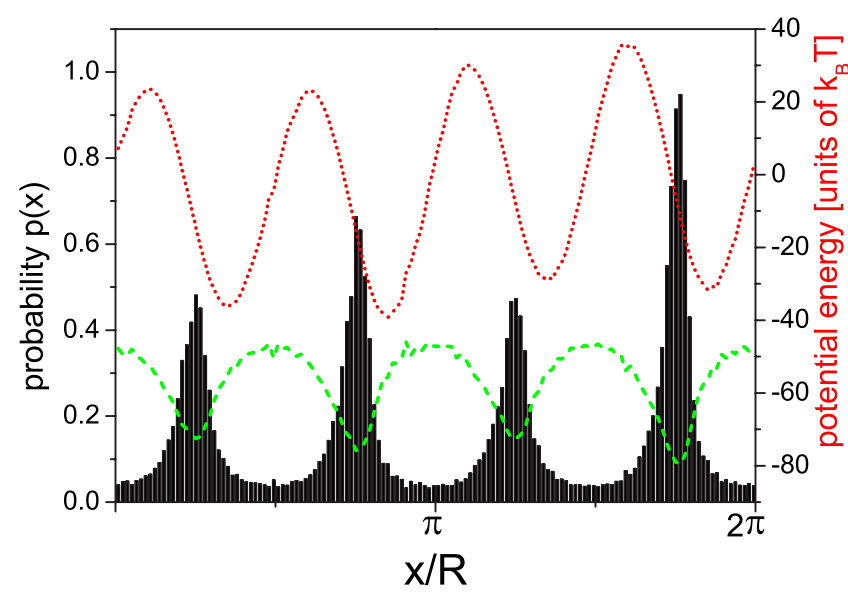

FIG. 1. (Color online) Stationary probability distribution $p_{\mathrm{s}}(x)$ (black bars) and the pseudopotential $\phi(x)$ (dashed line), measured at a driving force of $34 k_{\mathrm{B}} T / \mu \mathrm{m}$, pointing along the negative $x$ direction. The potential $V(x)$ (dotted line) is determined according to Eq. (6). Note that $\phi(x)$ is multiplied by a factor of 10 and shifted vertically to enhance visualization.

because $p_{\mathrm{s}}(x)$ is broader than it would be in equilibrium. On top of the intensity modulation according to Eq. (8) we observe a constant, small variation of the potential with $2 \pi$ periodicity caused by minute optical distortions in our setup. Since we are interested only in the local shape of the potential, in the following we only consider potentials where $V(x)$ is averaged over the four externally applied periods. The averaged potential, which is plotted as solid circles in Fig. 2, is in excellent agreement with a sinusoidal fit to

$$
V(x)=\frac{V_{0}}{2} \sin \left(4 \frac{2 \pi x}{L}\right)
$$

as theoretically expected for the optical potential in the case of sinusoidal intensity variations [16].

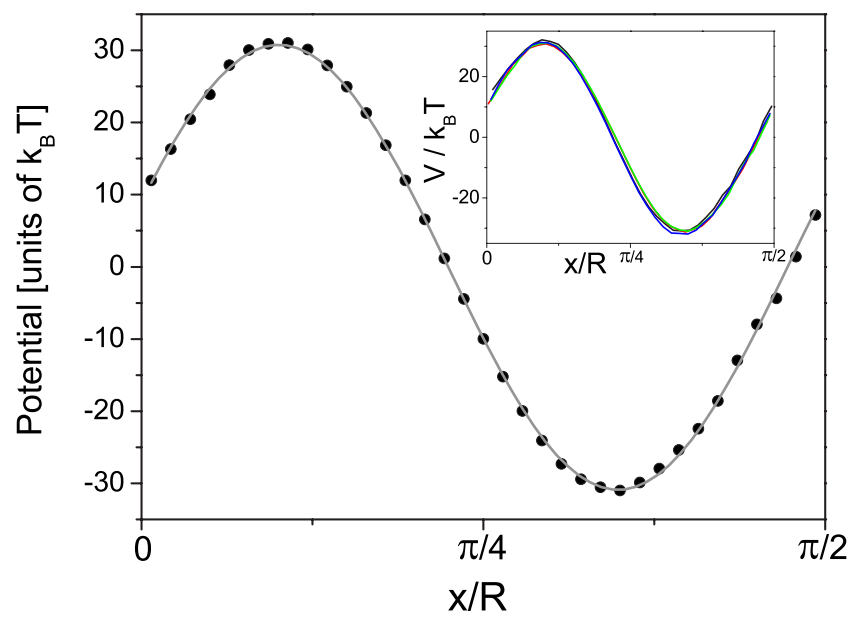

FIG. 2. (Color online) Averaged potential (solid points). The gray curve is a fit of Eq. (9) to the averaged potential. Inset: averaged potentials obtained for driving forces $f(=34,43,57$, and $\left.73 k_{\mathrm{B}} T / \mu \mathrm{m}\right)$. 


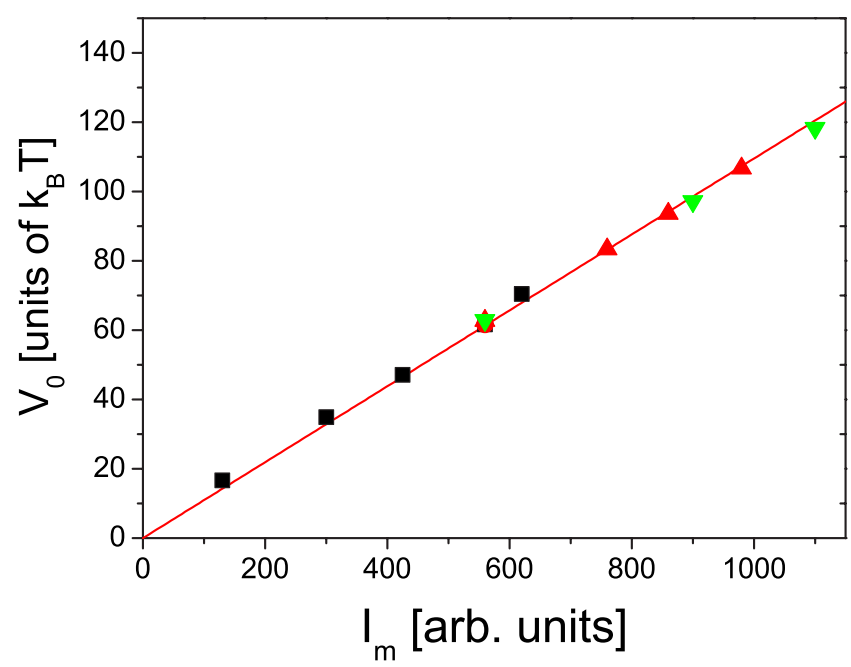

FIG. 3. (Color online) Potential depth $V_{0}$ as a function of the modulation amplitude $I_{m}$. The different symbols correspond to data acquired at different driving forces $\left(\boldsymbol{\square}, 43 k_{\mathrm{B}} T / \mu \mathrm{m}\right.$; $57 k_{\mathrm{B}} T / \mu \mathrm{m}$; and $\left.\boldsymbol{\nabla}, 73 k_{\mathrm{B}} T / \mu \mathrm{m}\right)$

To demonstrate the robustness of our approach in characterizing equilibrium potentials under NESS conditions, we systematically varied the driving force $f=34,43,57$, and $73 k_{\mathrm{B}} T / \mu \mathrm{m}$ while keeping $V(x)$ unchanged. Experimentally, this is achieved by changing $I_{0}$ with all other parameters in Eq. (8) fixed. The measured potentials plotted in the inset of Fig. 2 clearly fall on top of each other and thus demonstrate that the measured $V(x)$ is independent of $f$.

Similarly, the potential amplitude $V_{0}$ can be changed by variation of $I_{m}$. Figure 3 shows the potential depth as a function of $I_{m}$, measured at different driving forces $f$ (marked by different symbols). As expected, we find a linear dependence between $V_{0}$ and $I_{m}$ independent of $f$. This shows that reconstruction of equilibrium potentials can be reliably performed for a wide range of driving forces. For practical purposes, however, the driving force should not exceed a certain range where the lower limit is reached when the particle only rarely surmounts adjacent potential barriers and thus cannot sample the entire landscape. For very large $f$, the probability distribution becomes rather flat and very long sampling times are required to accurately measure $V(x)$.

Diffusion in tilted periodic potentials. Having demonstrated the validity of our approach to reconstruct equilibrium potentials under NESS conditions, in the following we will exemplarily apply this method to the problem of giant diffusion. It has been shown theoretically [17] and experimentally $[18,19]$ that the effective diffusion coefficient $D_{\text {eff }}$ of a Brownian particle moving in a tilted periodic potential $U(x)=V(x)+f x$ exhibits a pronounced maximum as a function of the driving force $f$. Until now experiments were not able to quantitatively match the theoretical predictions. With the ability to characterize the underlying potential landscape in detail, we can quantitatively test the theoretical behavior of $D_{\text {eff }}$.

The effective diffusion coefficient is easily obtained from the particle trajectory according to

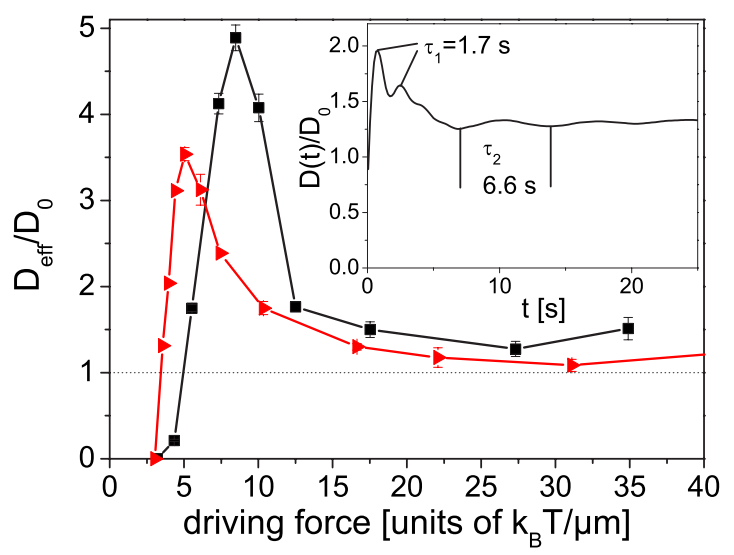

FIG. 4. (Color online) Normalized diffusion coefficient $D_{\text {eff }} / D_{0}$ vs external driving force. The data were obtained for potential depths $V_{0}$ of $10.3 k_{\mathrm{B}} T(\nabla)$ and $14.4 k_{\mathrm{B}} T(\boldsymbol{\square})$. Inset: the function $D(t)$ in Eq. (10) versus time $t\left(f \simeq 6.6 \mathrm{kT} / \mu \mathrm{m}, V_{0} \simeq 10.3 k_{\mathrm{B}} T\right)$. After $15 \mathrm{~s}$ the long-time limit is reached. The remaining small oscillations define the error in determining $D_{\text {eff }}$.

$$
D_{\text {eff }}=\lim _{t \rightarrow \infty} D(t), \quad D(t)=\frac{\left\langle x(t)^{2}\right\rangle-\langle x(t)\rangle^{2}}{2 t}
$$

This expression takes into account both the thermal diffusion and the drift motion evoked by the tilt of the potential. It is therefore applicable to both equilibrium and nonequilibrium conditions. Depending on the strength of the driving force, three regimes can be distinguished: (i) At small $f$, the particle is largely confined to the potential $V(x)$. Thus $D_{\text {eff }}<D_{0}$, where $D_{0}$, is the diffusion coefficient of a free particle. (ii) Around a critical force $f_{\mathrm{c}}$, where the local minima in the tilted potential vanish, a considerable enhancement of the thermal diffusion occurs-i.e., $D_{\text {eff }}>D_{0}$ [17]. (iii) In the limit of very large $f$ the potential becomes irrelevant and $D_{\text {eff }}$ eventually approaches $D_{0}$.

Our results are shown in Fig. 4, where we have chosen the same sinusoidal potential as above [see Eq. (9)] with typical amplitudes between $10 k_{\mathrm{B}} T$ and $20 k_{\mathrm{B}} T$ [20]. Since the infinite time limit required to calculate $D_{\text {eff }}$ cannot be realized in experiments, we first plotted the right-hand side of Eq. (10) as a function of time to determine when this expression saturates. The inset of Fig. 4 shows the result obtained for $f$ $\simeq 6.6 \mathrm{kT} / \mu \mathrm{m}$ and $V_{0} \simeq 10.3 k_{\mathrm{B}} T$. After an initial peak, the curve converges to the corresponding long-time value. A closer inspection reveals two damped oscillations whose periods are easily explained: The short oscillation time $\tau_{1}$ $\simeq 1.7$ s corresponds to the mean residence time of the particle within one minimum while the other oscillation with $\tau_{2} \simeq 6.6 \mathrm{~s} \simeq 4 \tau_{1}$ equals the mean revolution time of the particle along the torus. After about $t \gtrsim 15 \mathrm{~s}$, both oscillations have essentially decayed to the long-time value corresponding to $D_{\text {eff }}$.

Figure 4 shows the normalized effective diffusion coefficients for potential depths of $10.3 k_{\mathrm{B}} T$ and $14.4 k_{\mathrm{B}} T$. Both curves show a peak clearly indicating the enhancement of thermal diffusion in tilted periodic potentials. With increasing potential strength we observe a shift of the $D_{\text {eff }}(f)$ curve 
TABLE I. Comparison between experimentally determined and theoretically predicted positions and heights of the giant diffusion peak.

\begin{tabular}{lcccc}
\hline \hline & \multicolumn{2}{c}{$f_{c}\left[k_{\mathrm{B}} T / \mu \mathrm{m}\right]$} & \multicolumn{2}{c}{$D / D_{0}$} \\
\cline { 2 - 5 }$V_{0}\left[k_{\mathrm{B}} T\right]$ & Expt. & Theory & Expt. & Theory \\
\hline 14.4 & 8.5 & 7.3 & 4.9 & 3.1 \\
10.3 & 5.1 & 5.2 & 3.5 & 2.5 \\
\hline \hline
\end{tabular}

toward larger forces. The values of $f_{\mathrm{c}}$ and $D_{\text {eff }}\left(f_{\mathrm{c}}\right)$ sensitively depend on the shape of the potential. For our sinusoidal potential, characterized through Eq. (6), the critical force and the effective diffusion coefficient are theoretically predicted as [17] $f_{\mathrm{c}}=2 V_{0} / R$ and

$$
D_{\text {eff }}\left(f_{\mathrm{c}}\right)=0.0696 D_{0}\left(\frac{2}{3} V_{0} \pi^{3}\right)^{(2 / 3)}
$$

A comparison with our data is shown in Table I. While the predicted critical force $f_{\mathrm{c}}$ is in rather good agreement with the experimental data, the theoretical values systematically underestimate $D_{\text {eff }}\left(f_{\mathrm{c}}\right) / D_{0}$ by a factor of about 0.7 . The origin of this discrepancy is the aforementioned optical distortion, which adds an additional small $2 \pi$-periodic oscillation to the potential created by the EOM. As a result $V(x)$ does not show a perfect fourfold symmetry along the torus but slightly varies along the $x$ coordinate, causing small differences between the four potential minima. An estimate yields that the critical force, which is the global maximum in the potential slope, is enhanced by about $10 \%$. The effect of distortions in $V(x)$ on $D_{\text {eff }}$ is different. Equation (11) is based on a strictly periodic potential with only one point of inflection with positive slope [17], which cannot be directly compared to our experimental situation. On a qualitative level, the systematic underestimation of $D_{\text {eff }}$ can be understood as follows: If $f$ is close to $f_{\mathrm{c}}$, only one minimum in the tilted potential is left. Every time the particle surmounts this barrier by thermal fluctuations, it will drift across the whole circle before it experiences another barrier. This is in contrast to the fourfold-symmetric situation, where the bead has to overcome four barriers along one cycle. This effect leads to a larger diffusion coefficient and explains the underestimation of $D_{\text {eff }}$, when comparing our data with Eq. (11).

Concluding perspective. So far, we have demonstrated a method to reconstruct equilibrium potentials on the basis of the stationary probability distribution. In particular, in onedimensional NESS conditions, this quantity is easily determined experimentally, because the stationary current $j_{\mathrm{s}}$ is constant. When the method is extended to higher dimensions in the presence of nonconservative force fields $\mathbf{f}(\mathbf{r})$, in addition to the steady-state probability $p_{\mathrm{s}}(\mathbf{r})$, the local mean velocity $\mathbf{v}_{\mathrm{s}}(\mathbf{r})=\mathbf{j}_{\mathrm{s}}(\mathbf{r}) / p_{\mathrm{s}}(\mathbf{r})$ is required. Experimentally, this quantity is obtained by averaging the velocity of particles passing $\mathbf{r}$. Then the actual potential could be reconstructed through integration along open paths $C(\mathbf{r})$ starting at an arbitrary but fixed initial point and ending in $\mathbf{r}$, leading to

$$
V(\mathbf{r})=-k_{\mathrm{B}} T \ln p_{\mathrm{s}}(\mathbf{r})+\int_{C(\mathbf{r})}\left[\mathbf{f}(\mathbf{r}(s))-\gamma \mathbf{v}_{\mathrm{s}}(\mathbf{r}(s))\right] \cdot d \mathbf{r}(s) .
$$

In summary, we have demonstrated a flexible method to characterize potentials using the generalization of the inverted Boltzmann factor. In contrast to equilibrium measurements, this allows us to characterize laser potentials up to depths of several hundreds or even thousands of $k_{\mathrm{B}} T$. Based on the determination of the stationary-state probability distribution $p_{\mathrm{s}}$, this technique is easily applicable to different situations-e.g., topographical potentials-and does not require fast data acquisition techniques.
[1] A. Mehta, M. Rief, J. Spudich, D. Smith, and R. Simmons, Science 283, 1689 (1999).

[2] M. Wang, H. Yin, R. Landick, J. Gelles, and S. Block, Biophys. J. 72, 1335 (1997).

[3] A. Ehrlicher, T. Betz, B. Stuhrmann, D. Koch, V. Milner, M. G. Raizen, and J. Käs, Proc. Natl. Acad. Sci. U.S.A. 99, 16024 (2002).

[4] K. Mangold, P. Leiderer, and C. Bechinger, Phys. Rev. Lett. 90, 158302 (2003).

[5] M. Brunner and C. Bechinger, Phys. Rev. Lett. 88, 248302 (2002).

[6] M. McDonald, G. Spakling, and K. Dholakia, Nature (London) 426, 421 (2003).

[7] K. Ladavac and D. Grier, Europhys. Lett. 70, 548 (2005).

[8] A. Rohrbach, C. Tischner, D. Neumayer, and E.-L. Florin, Rev. Sci. Instrum. 75, 2197 (2004).

[9] P. Poulin, V. Cabuil, and D. A. Weitz, Phys. Rev. Lett. 79, 4862 (1997).

[10] M. Skarabot, M. Ravnik, D. Babic, N. Osterman, I. Poberaj, S. Zumer, I. Musevic, A. Nych, U. Ognysta, and V. Nazarenko,
Phys. Rev. E 73, 021705 (2006).

[11] L. P. Faucheux, G. Stolovitzky, and A. Libchaber, Phys. Rev. E 51, 5239 (1995).

[12] M. P. Sheetz, Laser Tweezers in Cell Biology (Academic Press, San Diego, 1998).

[13] L. Ghislain, N. Switz, and W. Webb, Rev. Sci. Instrum. 65, 2762 (1994).

[14] T. Speck and U. Seifert, Europhys. Lett. 74, 391 (2006).

[15] C. Lutz, M. Reichert, H. Stark, and C. Bechinger, Europhys. Lett. 74, 719 (2006).

[16] T. Tlusty, A. Meller, and R. Bar-Ziv, Phys. Rev. Lett. 81, 1738 (1998).

[17] P. Reimann, C. Van den Broeck, H. Linke, P. Hänggi, J. M. Rubi, and A. Perez-Madrid, Phys. Rev. Lett. 87, 010602 (2001); Phys. Rev. E 65, 031104 (2002).

[18] S. H. Lee and D. G. Grier, Phys. Rev. Lett. 96, 190601 (2006).

[19] S. A. Tatarkova, W. Sibbett, and K. Dholakia, Phys. Rev. Lett. 91, 038101 (2003).

[20] Deeper potentials are experimentally harder to explore because they lead to a rather sharp peak in $D_{\text {eff }}(f)$. 